\title{
BOCHNER'S THEOREM FOR SEMIGROUPS: A COUNTEREXAMPLE
}

\author{
TORBEN MAACK BISGAARD
}

\section{Introduction}

Weil [10], Povzner [5], and Raikov [6] proved (almost simultaneously) that if $G$ is a locally compact abelian group, a function $\varphi: G \rightarrow \mathrm{C}$ admits an integral representation of the form

$$
\varphi(s)=\int_{\widehat{G}} \sigma(s) d \mu(\sigma), \quad s \in G
$$

for some bounded measure $\mu$ on the dual group $\widehat{G}$ if and only if $\varphi$ is continuous and positive definite in the sense that

$$
\sum_{j, k=1}^{n} c_{j} \overline{c_{k}} \varphi\left(s_{j}-s_{k}\right) \geq 0
$$

for every choice of $s_{1}, \ldots, s_{n} \in G$ and $c_{1}, \ldots, c_{n} \in \mathrm{C}$.

So far, no generalization to semigroups is known which is of the same simplicity and generality. However, a comprehensive theory of integral representations of positive definite functions on semigroups exists. For this subject, see Berg, Christensen, and Ressel [2] and Berg [1].

Suppose $(S,+, *)$ is an abelian semigroup with zero and involution. A function $\varphi: S \rightarrow \mathrm{C}$ is positive definite if

$$
\sum_{j, k=1}^{n} c_{j} \overline{c_{k}} \varphi\left(s_{j}+s_{k}^{*}\right) \geq 0
$$

for every choice of $s_{1}, \ldots, s_{n} \in S$ and $c_{1}, \ldots, c_{n} \in \mathrm{C}$. Denote by $\mathscr{P}(S)$ the set of all positive definite functions on $S$, and by $\mathscr{P}_{1}(S)$ the subset of those $\varphi \in \mathscr{P}(S)$ such that $\varphi(0)=1$.

A function $\sigma: S \rightarrow \mathrm{C}$ is a character if $\sigma(0)=1, \sigma\left(s^{*}\right)=\overline{\sigma(s)}$, and $\sigma(s+t)=$ $\sigma(s) \sigma(t)$ for all $s, t \in S$. The set $\widehat{S}$ of all bounded characters on $S$ is a compact 
involution semigroup when considered with pointwise multiplication and complex conjugation and the topology of pointwise convergence. The semigroup $\widehat{S}$ has the unit 1 (the constant character), and $|\sigma(s)| \leq 1$ for all $\sigma \in \widehat{S}$ and $s \in S$.

Denote by $\mathscr{K}(\widehat{S})$ the set of all compact subsets of $\widehat{S}$. A measure $\mu$ defined on the Borel $\sigma$-field $\mathscr{B}(\widehat{S})$ is a Radon measure if $\mu(B)=\sup \{\mu(C) \mid \mathscr{K}(\widehat{S}) \ni$ $C \subset B\}$ for each $B \in \mathscr{B}(\widehat{S})$. Denote by $M_{+}(\widehat{S})$ the set of all Radon measures on $\widehat{S}$, and by $M_{+}^{1}(\widehat{S})$ the subset of probability measures. For $\mu \in M_{+}(\widehat{S})$, define $\widehat{\mu}: S \rightarrow \mathrm{C}$ by

$$
\widehat{\mu}(s)=\int_{\widehat{S}} \sigma(s) d \mu(\sigma), \quad s \in S .
$$

By the Lindahl-Maserick Theorem [4], a function $\varphi: S \rightarrow \mathrm{C}$ is bounded and positive definite if and only if $\varphi=\widehat{\mu}$ for some $\mu \in M_{+}(\widehat{S})$, and when the condition holds, $\mu$ is unique.

Now suppose $S$ is equipped with a topology rendering addition and involution continuous. It might be hoped that if $\mu \in M_{+}(\widehat{S})$ were such that $\widehat{\mu}$ were continuous then $\mu$-almost all characters would be continuous.

Certainly there are some topological *-semigroups, which are not groups, but for which the analogue of Bochner's theorem holds in the "continuity everywhere" version. Thus Ross [9] obtained a Bochner-type theorem for the duals of totally ordered semilattices which are locally compact in the order topology. Also, for $S=\left(\mathrm{R}_{+}^{k},+, s^{*}=s\right)$, a Bochner-type theorem holds ([2], 4.4.7).

Suppose $X$ is a locally compact Hausdorff space and $(\mathscr{K}, \cup)$ is the semilattice of compact subsets of $X$. A function $\varphi: \mathscr{K} \rightarrow \mathrm{R}$ is continuous on the right if for each $K \in \mathscr{K}$ and each $\varepsilon>0$ there is an open neighbourhood $G$ of $K$ such that $|\varphi(K)-\varphi(L)|<\varepsilon$ for each $L \in \mathscr{K}$ satisfying $K \subset L \subset G$. The functions on $\mathscr{K}$ continuous on the right are the functions continuous for a certain topology on $\mathscr{K}$. As shown by Choquet [3] (cf. [2], $4.6 .15 \mathrm{ff}$.), if $\mu \in M_{+}(\widehat{\mathscr{K}})$ is such that $\widehat{\mu}$ is continuous on the right then $\mu$-almost all characters are continuous on the right.

Suppose $S$ is a subsemigroup of $\mathrm{R}^{k}$, containing 0 , which is conelike in the sense that for each $s \in S$ there is some $a \in \mathrm{R}_{+}$such that $\alpha s \in S$ for all $\alpha \geq a$. Ressel [7] proved that if either $S$ carries the identical involution and 0 is in the closure of the set of those $s \in S$ such that $\left\{\alpha s \mid \alpha \in \mathrm{R}_{+}\right\}$intersects the interior of $S$, or $S$ carries an involution inherited from $\mathrm{R}^{k}$ and 0 is in the closure of the interior of $S$, then Bochner's theorem holds in the "continuity everywhere" version. Moreover, in each case, continuity at 0 of a bounded positive definite function on $S$ implies continuity everywhere.

Berg, Christensen, and Ressel ([2], p. 143) pointed out that on the compact 
semilattice $([0,1], \vee)$ with the usual topology, the only continuous character is the constant 1 . On this semigroup, the function $s \mapsto 1-s$ is a bounded continuous positive definite function, the representing measure of which is concentrated on the set of discontinuous characters.

Call a function $\varphi: S \rightarrow \mathrm{C}$ 0-continuous if $\varphi$ is continuous at 0 . Berg, Christensen, and Ressel ([2], p. 143) suggested that the right dual object of a locally compact Hausdorff $*$-semigroup might be the set $S^{\prime}$ of bounded 0continuous characters. By "Bochner's Theorem" for $S$ we shall understand the statement (which may be true or false) that if $\mu \in M_{+}(\widehat{S})$ is such that $\widehat{\mu}$ is 0 -continuous then $\mu$-almost all characters are 0 -continuous.

We note that Bochner's Theorem holds for $S$ if $S$ is one of the conelike subsemigroups of $\mathrm{R}^{k}$ for which Ressel proved a "continuity everywhere" version of Bochner's theorem (see above).

Suppose $S$ is a topological abelian semigroup with zero and the identical involution. Call a subset $U$ of $S$ cornered if for all $u, v \in U$ there is some $w \in U$ such that $\sigma(w) \leq \min \{\sigma(u), \sigma(v)\}$ for all $\sigma \in \widehat{S}$. Say that $S$ has property $C$ if $S$ has a neighbourhood base at 0 consisting of cornered sets. Ressel [8] proved that Bochner's Theorem holds for every semigroup having property C. In particular, this is so for every topological semilattice having a neighbourhood base at 0 consisting of subsemigroups (hence for every totally ordered semilattice considered with the order topology). Property $\mathrm{C}$ is stable under finite products and under open continuous homomorphic images (openness and continuity only to be required locally at 0 ).

Although it might be hoped that Bochner's Theorem, as we have defined it, would hold for every locally compact Hausdorff $*$-semigroup, or at least in the compact metrizable case, we shall destroy this hope by establishing the following:

THEOREM 1. There is a compact metrizable semilattice which admits a continuous positive definite function represented by a measure supported by the set of 0-discontinuous characters.

The semilattice $S$ in our counterexample will be constructed in two steps: First, we define a compact semimetrizable but non-Hausdorff semilattice $T$ and an appropriate function on it, then we proceed to the metric quotient semilattice $S$.

As a semilattice, $T$ is simply the product of $\left(2^{\mathrm{N}}, \cup\right.$ ) (the semilattice of subsets of the natural numbers) and $([0,1], \vee)$ where $\vee$ is the maximum operation on $[0,1]$. The topology on $T$ is defined by choosing $\varphi \in \mathscr{P}(T)$ in a very special way and then referring to the following result:

Theorem 2. Suppose $(T, \cup)$ is a semilattice and $\varphi \in \mathscr{P}(T)$. The equation 


$$
d(s, t)=\varphi(s)+\varphi(t)-2 \varphi(s \cup t)
$$

defines a semimetric on $T$ which renders $\cup$ and $\varphi$ continuous.

\section{Preparations}

Suppose $(S, \cup)$ is a semilattice, that is, an abelian semigroup with zero satisfying $s \cup s=s$ for all $s \in S$ and considered with the identical involution. We consider $S$ with the partial ordering $\leq$ defined by the condition that $s \leq t$ if and only if $s \cup t=t$. If $\sigma \in \widehat{S}$ and $s \in S$ then $\sigma(s)^{2}=\sigma(s \cup s)=\sigma(s)$, so $\sigma$ is $\{0,1\}$-valued.

Suppose $\varphi \in \mathscr{P}(S)$. Then $\varphi$ is nonnegative since the definition of positive definiteness, with $n=1, s_{1}=s$, and $c_{1}=1$ yields $0 \leq \varphi(s \cup s)=\varphi(s)$. Moreover, $\varphi$ is nonincreasing since if $s \leq t$ then the definition of positive definiteness, with $n=2, s_{1}=s, s_{2}=t, c_{1}=1$, and $c_{2}=-1$, yields

$$
0 \leq \varphi(s \cup s)+\varphi(t \cup t)-2 \varphi(s \cup t)=\varphi(s)-\varphi(t) .
$$

In particular, $\varphi(s) \leq \varphi(0)$ for all $s \in S$, so $\varphi$ is bounded and therefore $\varphi=\widehat{\mu}$ for some $\mu \in M_{+}(\widehat{S})$. (If $S$ is totally ordered then conversely, every nonincreasing nonnegative function on $S$ is positive definite, cf. [2], 4.4.18.) Since every character on $S$ is $\{0,1\}$-valued,

$$
0 \leq \int_{\widehat{S}}(1-\sigma(s))(1-\sigma(t)) d \mu(\sigma)=\varphi(0)-\varphi(s)-\varphi(t)+\varphi(s \cup t)
$$

for $s, t \in S$. This inequality can be written in the form

$$
\varphi(0)-\varphi(s \cup t) \leq(\varphi(0)-\varphi(s))+(\varphi(0)-\varphi(t))
$$

from which, by induction, it follows that

$$
\varphi(0)-\varphi\left(s_{1} \cup \cdots \cup s_{n}\right) \leq \sum_{j=1}^{n}\left(\varphi(0)-\varphi\left(s_{j}\right)\right)
$$

for $s_{1}, \ldots, s_{n} \in S$.

Suppose $A$ is a set. Denote by $2^{A}$ (resp. $2^{(A)}$ ) the set of all subsets (resp. finite subsets) of $A$. Consider the semilattice $S=\left(2^{(A)}, \cup\right)$. If $Q \in 2^{A}$ then a character $\sigma$ on $S$ is defined by the condition that $\sigma(P)=1$ if and only if $P \subset Q$. In this way, $\widehat{S}$ is identified with $2^{A}$, and for $\mu \in M_{+}\left(2^{A}\right)$ and $P \in S$ we write

$$
\widehat{\mu}(P)=\mu\left(\left\{Q \in 2^{A} \mid P \subset Q\right\}\right) .
$$

Lemma 1. Suppose $S$ is a topological abelian semigroup with zero and involution and $\varphi, \omega \in \mathscr{P}(S)$. If $\varphi+\omega$ is continuous at 0 , so are $\varphi$ and $\omega$. 
Proof. The fact that $\varphi \in \mathscr{P}(S)$ implies $|\varphi(s)|^{2} \leq \varphi(0) \varphi\left(s+s^{*}\right)$ for $s \in S$. Hence, if $\varphi(0)=0$ then $\varphi$ vanishes identically. Thus we may assume $\varphi(0), \omega(0)>0$. Now

$$
\begin{aligned}
\frac{|\varphi(0)-\varphi(s)|^{2}}{\varphi(0)} & +\frac{|\omega(0)-\omega(s)|^{2}}{\omega(0)} \leq\left[\varphi(0)-\varphi(s)-\varphi\left(s^{*}\right)+\varphi\left(s+s^{*}\right)\right] \\
& +\left[\omega(0)-\omega(s)-\omega\left(s^{*}\right)+\omega\left(s+s^{*}\right)\right],
\end{aligned}
$$

and the right-hand side tends to 0 as $s \rightarrow 0$.

Proposition 1. For a topological abelian semigroup $S$ with zero and involution, the following two conditions are equivalent:

(i) Bochner's Theorem holds for $S$;

(ii) if $K$ is a compact subset of $\widehat{S} \backslash S^{\prime}$ and if $\mu \in M_{+}^{1}(K)$ then $\widehat{\mu}$ is discontinuous at 0 .

Proof. Suppose (i) does not hold. Choose $\lambda \in M_{+}(\widehat{S})$ such that $\hat{\lambda}$ is 0 continuous, yet the inner measure $\lambda_{*}\left(\widehat{S} \backslash S^{\prime}\right)$ is positive. Choose a Borel set $A \subset \widehat{S} \backslash S^{\prime}$ such that $\lambda(A)>0$, then choose a compact set $K \subset A$ such that $\lambda(K)>0$. If $\varphi=(\lambda \mid \widehat{S} \backslash K)^{\wedge}$ and $\omega=(\lambda \mid K)^{\wedge}$ then $\varphi+\omega=\widehat{\lambda}$, so $\varphi$ and $\omega$ are 0 -continuous by Lemma 1 . Now $\mu=(\lambda \mid K) / \lambda(K)$ defines a probability measure $\mu$ on $K$ with $\widehat{\mu} 0$-continuous, so (ii) does not hold. Thus (ii) implies (i). It is trivial that (i) implies (ii).

Proposition 2. Suppose $(S, \cup)$ is a first countable topological semilattice. If $K$ is a compact subset of $\widehat{S} \backslash S^{\prime}$, there is a sequence $\left(a_{n}\right)_{n=1}^{\infty}$, converging to 0 in $S$, such that $\sigma\left(a_{n}\right)=0$ for infinitely many $n$ for each $\sigma \in K$.

PROoF. Let $\left(A_{n}\right)_{n=1}^{\infty}$ be a neighbourhood base at 0 with $A_{1} \supset A_{2} \supset \cdots$. For $\sigma \in K$, choose $b_{\sigma} \in A_{1}$ such that $\sigma\left(b_{\sigma}\right)=0$. The set $G_{\sigma}=\left\{\rho \in \widehat{S} \mid \rho\left(b_{\sigma}\right)=0\right\}$ is a neighbourhood of $\sigma$. Since $K$ is compact, there exist $\sigma_{1}, \ldots, \sigma_{n_{1}} \in K$ such that $K \subset \bigcup_{i=1}^{n_{1}} G_{\sigma_{i}}$. With $a_{i}=b_{\sigma_{i}}$ we have $a_{1}, \ldots, a_{n_{1}} \in A_{1}$, and for each $\sigma \in K$ there is some $i \in\left\{1, \ldots, n_{1}\right\}$ such that $\sigma\left(a_{i}\right)=0$. Similarly, choose $a_{n_{1}+1}, \ldots, a_{n_{1}+n_{2}} \in A_{2}$ such that for each $\sigma \in K$ there is some $i \in\left\{1, \ldots, n_{2}\right\}$ such that $\sigma\left(a_{n_{1}+i}\right)=0$. Continuing in this way, we get a sequence $\left(a_{n}\right)$ with the desired property.

Suppose $(S, \cup)$ is a metrizable semilattice for which Bochner's Theorem fails. By Proposition 1, there exist a compact subset $K$ of $\widehat{S} \backslash S^{\prime}$ and a probability measure $\mu$ on $K$ such that $\widehat{\mu}$ is continuous at 0 . By Proposition 2, there is a sequence $\left(a_{n}\right)_{n=1}^{\infty}$, converging to 0 in $S$, such that $\sigma\left(a_{n}\right)=0$ for infinitely many $n$ for each $\sigma \in K$. Denote by $T$ the semilattice $\left(2^{(\mathrm{N})}, \cup\right)$. Define a homomorphism $a: T \rightarrow S$ by 


$$
a(P)=\sum_{p \in P} a_{p}, \quad P \in T .
$$

Consider $T$ with the initial topology associated with the mapping $a$. Then $\{p\} \rightarrow \emptyset$ in $T$ as $N \ni p \rightarrow \infty$. Define a continuous mapping $\widehat{a}: \widehat{S} \rightarrow \widehat{T}$ by $\widehat{a}(\sigma)=\sigma \circ a, \sigma \in \widehat{S}$. Let $\nu$ be the probability measure on $\widehat{T}$ defined by $\nu=\mu^{a}$ (the image of $\mu$ under the mapping $\widehat{a}$ ). With $L=\widehat{a}(K)$, we have $L \subset \widehat{T} \backslash T^{\prime}, L$ is compact, $\nu$ is supported by $L$, and the function $\widehat{\nu}=\widehat{\mu} \circ a$ is continuous at 0 . Thus, every example of a metrizable semilattice for which Bochner's Theorem fails gives rise to an example in which the semigroup is $\left(2^{(\mathrm{N})}, \cup\right)$ equipped with a semimetric such that $\{p\} \rightarrow \emptyset$ as $p \rightarrow \infty$.

Proof of Theorem 2. Let $\mu \in M_{+}(\widehat{S})$ be the unique measure such that $\varphi=\widehat{\mu}$. Writing $K_{s}=\{\sigma \in \widehat{T} \mid \sigma(s)=1\}$ for $s \in T$, we have $\varphi(s)=\mu\left(K_{s}\right)$ and $\varphi(s \cup t)=\mu\left(K_{s} \cap K_{t}\right)$ for $s, t \in T$. Now $\varphi(s)-\varphi(s \cup t)=\mu_{s}\left(K_{s} \backslash K_{t}\right)$ and similarly for $\varphi(t)-\varphi(s \cup t)$, so

$$
d(s, t)=\mu\left(K_{s} \triangle K_{t}\right), \quad s, t \in T
$$

with the notation $L \triangle M=(L \backslash M) \cup(M \backslash L)$. For $s, t, u \in T$ we have $K_{s} \backslash K_{u} \subset\left(K_{s} \backslash K_{t}\right) \cup\left(K_{t} \backslash K_{u}\right)$ and similarly for $K_{u} \backslash K_{s}$, so $K_{s} \triangle K_{u} \subset\left(K_{s} \triangle K_{t}\right) \cup\left(K_{t} \triangle K_{u}\right)$ and therefore $d(s, u) \leq d(s, t)+d(t, u)$, which shows that $d$ is a semimetric. For $s, t, u, v \in T$ we have

$$
\begin{aligned}
& \left(K_{s} \cap K_{u}\right) \backslash\left(K_{t} \cap K_{v}\right) \\
& =\left(\left(K_{s} \cap K_{u}\right) \backslash K_{t}\right) \cup\left(\left(K_{s} \cap K_{u}\right) \backslash K_{v}\right) \subset\left(K_{s} \backslash K_{t}\right) \cup\left(K_{u} \backslash K_{v}\right)
\end{aligned}
$$

and similarly for $\left(K_{t} \cap K_{v}\right) \backslash\left(K_{s} \cap K_{u}\right)$, so $\left(K_{s} \cap K_{u}\right) \triangle\left(K_{t} \cap K_{v}\right) \subset\left(K_{s} \triangle K_{t}\right) \cup$ $\left(K_{u} \triangle K_{v}\right)$ and therefore

$$
d(s \cup u, t \cup v) \leq d(s, t)+d(u, v), \quad s, t, u, v \in T
$$

which shows that $T$ is a topological semigroup. Finally, since $\varphi$ is nonincreasing then

$$
|\varphi(s)-\varphi(t)| \leq d(s, t), \quad s, t \in T
$$

which shows that $\varphi$ is continuous.

Recall that the space $\left(2^{(\mathrm{N})}, \cup\right)^{\wedge}$ is identified with $2^{\mathrm{N}}$.

Proposition 3. Suppose $\mu \in M_{+}^{1}\left(2^{\mathrm{N}}\right), n \notin Q$ for infinitely many $n$ for each $Q$ in the support of $\mu$, and define $\varphi=\widehat{\mu}$. If there is some semimetric on $2^{(\mathrm{N})}$ which makes the completion of $2^{(\mathrm{N})}$ a compact topological semigroup admitting a continuous extension of $\varphi$, and which satisfies $\{n\} \rightarrow \emptyset$ as $n \rightarrow \infty$, then the function $d$ defined by $d(P, Q)=\varphi(P)+\varphi(Q)-2 \varphi(P \cup Q)$ is such a semimetric. 
Proof. Suppose $e$ is a semimetric satisfying the assumptions, and define $d$ as in the statement. Every sequence in $2^{(\mathrm{N})}$ has a subsequence $\left(P_{n}\right)$ such that $P_{n} \rightarrow \Pi$ for some $\Pi$ in the completion of $2^{(\mathrm{N})}$ with respect to $e$, so $P_{m} \cup P_{n} \rightarrow \Pi$ and therefore $\varphi\left(P_{n}\right) \rightarrow \varphi(\Pi)$ and $\varphi\left(P_{m} \cup P_{n}\right) \rightarrow \varphi(\Pi)$ as $m, n \rightarrow \infty$ (denoting by $\varphi$ also the continuous extension of $\varphi$ on the completion of $2^{(\mathrm{N})}$ with respect to $e$ ). Thus $d\left(P_{m}, P_{n}\right) \rightarrow 0$ as $m, n \rightarrow \infty$, which shows that the completion of $2^{(\mathrm{N})}$ with respect to $d$ is compact. The inequality (5) shows that $\varphi$ extends to a continuous function on the completion of $2^{(\mathrm{N})}$ with respect to $d$. Finally, since $\{n\} \rightarrow \emptyset$ with respect to $e$ as $n \rightarrow \infty$, and since $\varphi$ is continuous with respect to $e$, then $d(\emptyset,\{n\})=\varphi(\emptyset)-\varphi(\{n\}) \rightarrow 0$.

Lemma 2. Suppose $K$ is a compact subset of $2^{\mathrm{N}}$ such that $p \notin Q$ for infinitely many $p$ for all $Q \in K$. Suppose $\mu \in M_{+}^{1}\left(2^{\mathrm{N}}\right)$ and define $\varphi=\widehat{\mu}$. Then there is a sequence $\left(N_{n}\right)_{n=1}^{\infty}$ of positive integers such that with

$$
A_{n}=\left\{N_{1}+\cdots+N_{n-1}+1, \ldots, N_{1}+\cdots+N_{n}\right\}, \quad n \in \mathrm{N}
$$

we have $\varphi\left(A_{n}\right)=0$ for all $n$. If $\varphi(\{p\}) \rightarrow 1$ as $p \rightarrow \infty$ then $N_{n} \rightarrow \infty$ as $n \rightarrow \infty$.

Proof. The set $\left\{\left\{Q \in 2^{\mathrm{N}} \mid p \notin Q\right\} \mid p \in \mathrm{N}\right\}$ is an open covering of $K$, so we can choose $N_{1}$ such that $\varphi\left(A_{1}\right)=0$. Choose $N_{2}, N_{3}, \ldots$ similarly. If $\varphi(\{p\}) \rightarrow 1$ as $p \rightarrow \infty$ then from

$$
1=1-\varphi\left(A_{n}\right) \leq \sum_{p \in A_{n}}(1-\varphi(\{p\})) \leq N_{n} \sup \left\{1-\varphi(\{p\}) \mid p \in A_{n}\right\}
$$

(cf. (3)) we get $N_{n} \rightarrow \infty$.

In the following, suppose that $\left(N_{n}\right)_{n=1}^{\infty}$ is a sequence of positive integers, and define a partition $\left(A_{n}\right)_{n=1}^{\infty}$ of $\mathrm{N}$ by (6). We plan to choose $\varphi_{n} \in \mathscr{P}_{1}\left(2^{\left(A_{n}\right)}\right)$ suitably and then extend the $\varphi_{n}$ to a positive definite function $\varphi$ on $2^{(\mathrm{N})}$, in order to equip $2^{(\mathrm{N})}$ with the semimetric $d$ defined as in Theorem 2 and proceed to the completion of $2^{(\mathrm{N})}$. One method of extending the $\varphi_{n}$ to a positive definite function on $2^{(\mathrm{N})}$ is to define

$$
\varphi(P)=\prod_{n=1}^{\infty} \varphi_{n}\left(P \cap A_{n}\right), \quad P \in 2^{(\mathrm{N})} .
$$

In terms of the measures $\mu_{n} \in M_{+}^{1}\left(2^{A_{n}}\right)$ and $\mu \in M_{+}^{1}\left(2^{\mathrm{N}}\right)$ such that $\varphi_{n}=\widehat{\mu}_{n}$ and $\varphi=\widehat{\mu}$, this would correspond to taking $\mu$ to be the product measure $\mu=\bigotimes_{n=1}^{\infty} \mu_{n}$. This, however, would not do, as we shall see next.

Say that a function $\varphi: 2^{(\mathrm{N})} \rightarrow[0,1]$ has the compactness property if every sequence in $2^{(\mathrm{N})}$ has a subsequence $\left(P_{i}\right)$ such that for some $s \in[0,1]$ we have $\varphi\left(P_{i}\right) \rightarrow s$ and $\varphi\left(P_{i} \cup P_{j}\right) \rightarrow s$ as $i, j \rightarrow \infty$. If $\varphi \in \mathscr{P}_{1}\left(2^{(\mathrm{N})}\right)$ then $\varphi$ has the compactness property if and only if the completion of $2^{(\mathrm{N})}$ with respect to 
the semimetric $d$ defined as in Theorem 2 is compact. Indeed, by the definition of $d$, a sequence $\left(P_{i}\right)$ is a Cauchy sequence if and only if $\varphi\left(P_{i}\right)-\varphi\left(P_{i} \cup P_{j}\right) \rightarrow 0$ as $i, j \rightarrow \infty$.

Lemma 3. For $n \in \mathrm{N}$, suppose $\mu_{n} \in M_{+}^{1}\left(2^{A_{n}}\right)$. Define $\mu=\bigotimes_{n=1}^{\infty} \mu_{n} \in M_{+}^{1}\left(2^{\mathrm{N}}\right)$ and $\varphi=\widehat{\mu} \in \mathscr{P}_{1}\left(2^{(\mathrm{N})}\right)$. Suppose $\varphi\left(A_{n}\right)=0$ for all $n \in \mathrm{N}$. If $\varphi(\{p\}) \rightarrow 1$ as $p \rightarrow \infty$ then $\varphi$ does not have the compactness property.

Proof. Suppose $\varphi(\{p\}) \rightarrow 1$ as $p \rightarrow \infty$. Choose $n_{0} \in \mathbf{N}$ such that $1-\varphi(\{p\})<1 / 6$ for $p \in A_{n}$ with $n \geq n_{0}$. Define

$$
B_{n}^{m}=\left\{N_{1}+\cdots+N_{n-1}+1, \ldots, N_{1}+\cdots+N_{n-1}+m\right\}
$$

for $n \geq n_{0}$ and $m=0, \ldots, N_{n}$. Then $\varphi\left(B_{n}^{0}\right)=1, \varphi\left(B_{n}^{N_{n}}\right)=0$, and by (3),

$$
\varphi\left(B_{n}^{m-1}\right)-\varphi\left(B_{n}^{m}\right) \leq 1-\varphi\left(\left\{N_{1}+\cdots+N_{n-1}+m\right\}\right)<\frac{1}{6},
$$

so there is some $m_{n} \in\left\{0, \ldots, N_{n}\right\}$ such that $1 / 2<\varphi\left(C_{n}\right)<2 / 3$ where $C_{n}=B_{n}^{m_{n}}$. Then $\varphi\left(C_{i} \cup C_{j}\right)=\varphi\left(C_{i}\right) \varphi\left(C_{j}\right)<4 / 9$ for $i \neq j$, so no subsequence of $\left(C_{n}\right)$ has the property required in the definition of the compactness property.

We thus have to find a measure $\mu$ on $2^{\mathrm{N}}$ with the marginals $\mu_{n}$ with respect to the natural identification of $2^{\mathrm{N}}$ with $\prod_{n=1}^{\infty} 2^{A_{n}}$, and distinct from the product measure. For $n \in \mathrm{N}$ and $k=0, \ldots, N_{n}$, define $\Omega_{n, k}=\left\{Q \in 2^{A_{n}} \mid\right.$. $|Q|=k\}$. Then

$$
2^{A_{n}}=\bigcup_{k=0}^{N_{n}} \Omega_{n, k},
$$

so there exist $b_{n, 0}, \ldots, b_{n, N_{n}} \geq 0$ and measures $\mu_{n, 0} \in M_{+}^{1}\left(\Omega_{n, 0}\right), \ldots, \mu_{n, N_{n}} \in$ $M_{+}^{1}\left(\Omega_{n, N_{n}}\right)$ such that

$$
\mu_{n}=\sum_{k=0}^{N_{n}} b_{n, k} \mu_{n, k}
$$

For $0 \leq t \leq 1$, define $\mu_{t, n} \in M_{+}^{1}\left(2^{A_{n}}\right)$ by the condition that

$$
b_{n, 0}+\cdots+b_{n, k-1} \leq t<b_{n, 0}+\cdots+b_{n, k} \Rightarrow \mu_{t, n}=\mu_{n, k}
$$

together with $\mu_{1, n}=\mu_{n, N_{n}}$. Then

$$
\mu_{n}=\int_{0}^{1} \mu_{t, n} d t
$$

in the sense that $\int f d \mu_{n}=\int_{0}^{1} \int f d \mu_{t, n} d t$ for every function $f$ on $2^{A_{n}}$. Now define 


$$
\mu_{t}=\bigotimes_{n=1}^{\infty} \mu_{t, n} \in M_{+}^{1}\left(2^{\mathrm{N}}\right), \quad 0 \leq t \leq 1
$$

and

$$
\mu=\int_{0}^{1} \mu_{t} d t
$$

in the sense that $\int f d \mu=\int_{0}^{1} \int f d \mu_{t} d t$ for every continuous function $f$ on $2^{\mathrm{N}}$. Then $\mu$ has the marginals $\mu_{n}$.

Instead of specifying the numbers $b_{n, 0}, \ldots, b_{n, N_{n}}$, we can specify the function $k_{n}:[0,1] \rightarrow\left\{0, \ldots, N_{n}\right\}$ defined by

$$
b_{n, 0}+\cdots+b_{n, k-1} \leq t<b_{n, 0}+\cdots+b_{n, k} \Rightarrow k_{n}(t)=k
$$

and $k_{n}(1)=N_{n}$. Then

$$
\mu_{t, n}=\mu_{n, k_{n}(t)}, \quad 0 \leq t \leq 1
$$

Suppose that $\varphi_{n}(P)$, for $n \in \mathrm{N}$ and $P \in 2^{\left(A_{n}\right)}$, is a function of the cardinality of $P$ alone:

$$
\varphi_{n}(P)=\Phi_{n}(|P|)
$$

where $|P|$ is the cardinality of $P$. The condition that such a function $\Phi_{n}$ exist is equivalent to the condition that $\mu_{n}(\{Q\})$, for $Q \in 2^{A_{n}}$, depend on the cardinality of $Q$ alone, or

$$
\mu_{n, k}=\left(\begin{array}{c}
N_{n} \\
k
\end{array}\right)^{-1} \sum_{Q \in \Omega_{n, k}} \varepsilon_{Q}
$$

where $\varepsilon_{Q}$ denotes the Dirac measure at $Q$. Then

$$
\varphi_{n}(P)=\sum_{k=0}^{N_{n}} b_{n, k}\left(\begin{array}{c}
N_{n} \\
k
\end{array}\right)^{-1}\left(\begin{array}{c}
N_{n}-j \\
k-j
\end{array}\right)=\sum_{k=0}^{N_{n}} b_{n, k} \frac{k^{(j)}}{N_{n}^{(j)}}
$$

for $P \in 2^{\left(A_{n}\right)}$ with $|P|=j$, with the notation $p^{(q)}=p(p-1) \ldots(p-q+1)$ for $p, q \in \mathrm{N}_{0}$. If we define

$$
\varphi_{t, n}=\widehat{\mu}_{t, n}, \quad 0 \leq t \leq 1
$$

then

$$
\varphi_{t, n}(P)=\frac{k_{n}(t)^{(j)}}{N_{n}^{(j)}} \quad \text { for } P \in 2^{\left(A_{n}\right)} \text { with }|P|=j .
$$

In the following, let $T=\left(2^{\mathrm{N}} \times[0,1], \cup\right)$ be the semilattice defined by 
$(P, u) \cup(Q, v)=(P \cup Q, u \vee v)$ for all $(P, u)$ and $(Q, v)$, where $\vee$ denotes the maximum operation on $[0,1]$.

Proposition 4. For $n \in \mathrm{N}$, suppose $k_{n}:[0,1] \rightarrow\left\{0, \ldots, N_{n}\right\}$ is a nondecreasing function. Define

$$
\Phi_{t, n}(j)=\frac{k_{n}(t)^{(j)}}{N_{n}^{(j)}}, \quad \varphi_{t, n}(P)=\Phi_{t, n}(|P|)
$$

for $n \in \mathrm{N}, 0 \leq t \leq 1, j=0, \ldots, N_{n}$, and $P \in 2^{\left(A_{n}\right)}$, and

$$
\varphi_{t}(P)=\prod_{n=1}^{\infty} \varphi_{t, n}\left(P \cap A_{n}\right), \quad \varphi(P, u)=\int_{u}^{1} \varphi_{t}(P) d t
$$

for $0 \leq t \leq 1, \quad P \in 2^{\mathrm{N}}, \quad$ and $\quad 0 \leq u \leq 1$. Then $\varphi_{t, n} \in \mathscr{P}_{1}\left(2^{\left(A_{n}\right)}\right)$, hence $\varphi_{t} \in \mathscr{P}_{1}\left(2^{\mathrm{N}}\right)$, hence $\varphi \in \mathscr{P}_{1}(T)$.

Proof. With $\mu_{t, n}$ as above, we have $\varphi_{t, n}=\widehat{\mu}_{t, n} \in \mathscr{P}_{1}\left(2^{\left(A_{n}\right)}\right)$. For $n \in \mathrm{N}$ the pointwise product $P \mapsto \prod_{m=1}^{n} \varphi_{t, m}\left(P \cap A_{m}\right)$ is in $\mathscr{P}\left(2^{\mathrm{N}}\right)$. Hence so is the pointwise limit $P \mapsto \prod_{n=1}^{\infty} \varphi_{t, n}\left(P \cap A_{n}\right)$. For $t \in[0,1]$ the function $1_{[0, t]}$ is a character on $([0,1], \vee)$, hence positive definite, so $(P, u) \mapsto \varphi_{t}(P) 1_{[0, t]}(u)$ is in $\mathscr{P}(T)$. Hence so is

$$
(P, u) \mapsto \int_{0}^{1} \varphi_{t}(P) 1_{[0, t]}(u) d t=\varphi(P, u) .
$$

(Since $k_{n}$ is nondecreasing, so are $t \mapsto \Phi_{t, n}(j), t \mapsto \varphi_{t, n}(P)$, and $t \mapsto \varphi_{t}(P)$, so there is no measurability problem.)

We observe that the following formulas hold:

$$
\begin{gathered}
\varphi_{t}\left(P \cap \bigcup_{n=1}^{k} A_{n}\right)=\prod_{n=1}^{k} \varphi_{t, n}\left(P \cap A_{n}\right), \\
\varphi_{t}\left(P \cap \bigcup_{n=k+1}^{\infty} A_{n}\right)=\prod_{n=k+1}^{\infty} \varphi_{t, n}\left(P \cap A_{n}\right), \\
\varphi_{t}(P)=\varphi_{t}\left(P \cap \bigcup_{n=1}^{k} A_{n}\right) \varphi_{t}\left(P \cap \bigcup_{n=k+1}^{\infty} A_{n}\right) .
\end{gathered}
$$

Lemma 4. In order that $\varphi(\{p\}, 0) \rightarrow 1$ as $p \rightarrow \infty$, it is necessary and sufficient that $k_{n}(t) / N_{n} \rightarrow 1$ as $n \rightarrow \infty$ for $0<t \leq 1$.

Proof. For $p \in A_{n}$ we have 


$$
\varphi(\{p\}, 0)=\int_{0}^{1} \frac{k_{n}(t)}{N_{n}} d t .
$$

If $k_{n}(t) / N_{n} \rightarrow 1$ as $n \rightarrow \infty$ for $0<t \leq 1$ then $\varphi(\{p\}, 0) \rightarrow 1$ by bounded convergence. Conversely, if $\varphi(\{p\}, 0) \rightarrow 1$ as $p \rightarrow \infty$ then for $0<u \leq 1$, from

$$
\varphi(\{p\}, 0) \leq u \frac{k_{n}(u)}{N_{n}}+1-u=1-\left(1-\frac{k_{n}(u)}{N_{n}}\right) u
$$

we get $k_{n}(u) / N_{n} \rightarrow 1$.

With a notation different from one previously used, let $\mu \in M_{+}^{1}(\widehat{T})$ be the unique measure such that $\varphi=\widehat{\mu}$. Denote by $L$ the set of those $\rho \in \widehat{T}$ such that $\rho(\{p\}, 0)=0$ for infinitely many $p \in \mathbf{N}$.

Lemma 5. In order that $\mu(L)=1$, it suffices that $k_{n}(t)<N_{n}$ for all $n \in \mathrm{N}$ and $t \in[0,1]$.

Proof. Suppose the condition holds. Then

$$
\varphi_{t}\left(A_{n}\right)=\frac{k_{n}(t)^{\left(N_{n}\right)}}{N_{n} !}=0
$$

for all $n \in \mathrm{N}$ and $t \in[0,1]$, so

$$
\mu\left(\left\{\rho \in \widehat{T} \mid \rho\left(A_{n}, 0\right)=1\right\}\right)=\varphi\left(A_{n}, 0\right)=0,
$$

which shows $\mu\left(L_{n}\right)=1$ where $L_{n}$ is the set of those $\rho \in \widehat{T}$ such that $\rho(\{p\}, 0)=0$ for at least one $p \in A_{n}$. Since $\bigcap_{n=1}^{\infty} L_{n} \subset L$, it follows that $\mu(L)=1$.

Consider $T$ with the topology induced by the semimetric $d$ defined as in Theorem 2.

THEOREM 3. In order that $T$ be compact, it suffices that

$$
\frac{\log \left[N_{n} / k_{n}(v)\right]}{\log \left[N_{n} / k_{n}(u)\right]} \rightarrow 0 \quad \text { as } n \rightarrow \infty \text { for } 0 \leq u<v \leq 1 .
$$

Proof. Suppose the condition holds. Let a sequence $\left(P_{i}, z_{i}\right)$ in $T$ be given. Choose an increasing sequence $\left(i_{k}\right)_{k=1}^{\infty}$ in $\mathrm{N}$ such that

$$
P_{i_{k}} \cap A_{k}=P_{i_{l}} \cap A_{k}, \quad l \geq k .
$$

(This is possible because $P_{i} \cap A_{k}$ is always one of the finitely many subsets of $A_{k}$.) Define $P \in 2^{\mathrm{N}}$ by $P \cap A_{k}=P_{i_{k}} \cap A_{k}$ for $k \in \mathrm{N}$. Write 


$$
Q_{k}=P_{i_{k}} \cap \bigcup_{n=k+1}^{\infty} A_{n}, \quad k \in \mathrm{N} .
$$

Choose a countable dense set $U$ in $[0,1]$, then choose an infinite subset $K$ of $\mathrm{N}$ such that $\varphi_{t}\left(Q_{k}\right) \rightarrow s(t)$ as $K \ni k \rightarrow \infty$ for some $s(t) \in[0,1]$ for all $t \in U$. Since $t \mapsto \varphi_{t}(Q)$ is nondecreasing for each $Q$ then the function $s$ is nondecreasing. If $t \in[0,1]$ is such that $s$ extends to a continuous function on $U \cup\{t\}$ then $\varphi_{t}\left(Q_{k}\right) \rightarrow s(t)$ as $K \ni k \rightarrow \infty$ for the unique $s(t)$ that makes the extension continuous. The remaining points of $[0,1]$ form a countable set, so we can choose an infinite subset $M$ of $K$ such that $\varphi_{t}\left(Q_{k}\right) \rightarrow s(t)$ as $M \ni k \rightarrow \infty$ for some $s(t) \in[0,1]$ for all $t \in[0,1]$.

We claim that there is some $w \in[0,1]$ such that

$$
s(t)=0 \quad \text { if } t<w, \quad s(t)=1 \quad \text { if } t>w .
$$

The function $s$ being nondecreasing, this is trivial if $s(t) \in\{0,1\}$ for all $t \in[0,1]$. Thus we may assume that there is some $w \in[0,1]$ such that $0<s(w)<1$. The claim will then follow if we can show that the conditions $0 \leq u<v \leq 1$ and $Q \subset \bigcup_{n=k+1}^{\infty} A_{n}$ imply $\log \varphi_{v}(Q) \geq \varepsilon_{k} \log \varphi_{u}(Q)$ with $\varepsilon_{k} \rightarrow 0$ as $k \rightarrow \infty$. Since $\log \varphi_{t}(Q)=\sum_{n=k+1}^{\infty} \log \varphi_{t, n}\left(Q \cap A_{n}\right)$, it suffices to show

$$
\log \varphi_{v, n}(R) \geq \delta_{n} \log \varphi_{u, n}(R), \quad R \subset A_{n}
$$

with $\delta_{n} \rightarrow 0$ as $n \rightarrow \infty$, or $\log \Phi_{v, n}(j) \geq \delta_{n} \log \Phi_{u, n}(j)$ for $0 \leq j \leq N_{n}$. Since $\log \Phi_{t, n}(j)=\sum_{i=0}^{j-1} \log \left[\left(k_{n}(t)-i\right) /\left(N_{n}-i\right)\right]$, it suffices to show

$$
\log \frac{k_{n}(v)-i}{N_{n}-i} \geq \delta_{n} \frac{k_{n}(u)-i}{N_{n}-i}, \quad 0 \leq i<k_{n}(u) .
$$

In other words, with

$$
f_{n}(x)=\frac{\log \left[\left(N_{n}-x\right) /\left(k_{n}(v)-x\right)\right]}{\log \left[\left(N_{n}-x\right) /\left(k_{n}(u)-x\right)\right]}, \quad 0 \leq x<k_{n}(u),
$$

it suffices to show that $f_{n}(x) \rightarrow 0$, uniformly in $x$, as $n \rightarrow \infty$. We have $f_{n}(0) \rightarrow 0$ by hypothesis, so it suffices to show that each $f_{n}$ is nonincreasing. Computation shows

$$
f_{n}^{\prime}(x) \leq 0 \Leftrightarrow g_{n, x}\left(k_{n}(u)\right) \leq g_{n, x}\left(k_{n}(v)\right)
$$

where

$$
g_{n, x}(y)=\frac{\log \frac{N_{n}-x}{y-x}}{\frac{1}{y-x}-\frac{1}{N_{n}-x}}, \quad x<y<N_{n}
$$


so it suffices to show that $g_{n, x}$ is nondecreasing for all $n$ and $x$. Differentiation shows

$$
g_{n, x}^{\prime}(y) \geq 0 \Leftrightarrow \log \frac{N_{n}-x}{y-x} \geq 1-\frac{y-x}{N_{n}-x} .
$$

The latter inequality is automatically fulfilled. This proves (10).

Finally, choose an infinite subset $N$ of $M$ such that $z_{i_{k}} \rightarrow z$ as $N \ni k \rightarrow \infty$ for some $z \in[0,1]$. We claim that the sequence $\left(\left(P_{i_{k}}, z_{i_{k}}\right)\right)_{k \in N}$ converges to $(P, w \vee z)$. We have to show $d\left(\left(P_{i_{k}}, z_{i_{k}}\right),(P, w \vee z)\right) \rightarrow 0$ as $N \ni k \rightarrow \infty$, and we do this by showing $\varphi\left(P_{i_{k}}, z_{i_{k}}\right) \rightarrow \varphi(P, w \vee z)$ and $\varphi\left(P_{i_{k}} \cup P, z_{i_{k}} \vee w \vee z\right) \rightarrow$ $\varphi(P, w \vee z)$. Firstly,

$$
\varphi\left(P_{i_{k}}, z_{i_{k}}\right)=\int_{z_{i_{k}}}^{1} \varphi_{t}\left(P_{i_{k}}\right) d t \rightarrow \int_{z}^{1} \lim _{k} \varphi_{t}\left(P_{i_{k}}\right) d t,
$$

provided that the limit exists for almost all $t$, so it suffices to show

$$
\lim _{k} \varphi_{t}\left(P_{i_{k}}\right)= \begin{cases}0, & \mathrm{t}<\mathrm{w} \\ \varphi_{t}(P), & \mathrm{t}>\mathrm{w}\end{cases}
$$

But this follows from $\varphi_{t}\left(P_{i_{k}}\right)=\varphi_{t}\left(P \cap \bigcup_{n=1}^{k} A_{n}\right) \varphi_{t}\left(Q_{k}\right)$ because of (10), cf. (9). Secondly,

$$
\varphi\left(P_{i_{k}} \cup P, z_{i_{k}} \vee w \vee z\right) \rightarrow \int_{w \vee z}^{1} \lim _{k} \varphi_{t}\left(P_{i_{k}} \cup P\right) d t,
$$

provided that the limit exists for almost all $t$, so it suffices to show $\lim _{k} \varphi_{t}\left(P_{i_{k}} \cup P\right)=\varphi_{t}(P)$ for $t>w$. Now by (9),

$$
\begin{aligned}
0 & \leq \varphi_{t}(P)-\varphi_{t}\left(P_{i_{k}} \cup P\right)=\varphi_{t}\left(P \cap \bigcup_{n=1}^{k} A_{n}\right)\left[\varphi_{t}\left(P \cap \bigcup_{n=k+1}^{\infty} A_{n}\right)\right. \\
& \left.-\varphi_{t}\left(Q_{k} \cup\left(P \cap \bigcup_{n=k+1}^{\infty} A_{n}\right)\right)\right] \leq \varphi_{t}\left(P \cap \bigcup_{n=k+1}^{\infty} A_{n}\right) \\
& -\varphi_{t}\left(Q_{k} \cup\left(P \cap \bigcup_{n=k+1}^{\infty} A_{n}\right)\right) \leq 1-\varphi_{t}\left(Q_{k}\right)
\end{aligned}
$$

because of (2), and the right-hand side tends to 0 because of (10). 


\section{The counterexample}

Proof of Theorem 1. Define $N_{n}=3^{n}$ and

$$
k_{n}(t)=\left\lfloor N_{n} e^{\left.-e^{-n t}\right\rfloor}, \quad n \in \mathrm{N}, \quad t \in[0,1]\right.
$$

where $\lfloor x\rfloor$ denotes the greatest integer not exceeding $x$. Now define everything else as in Proposition 4. Consider $T$ with the semimetric $d$ defined as in Theorem 2. We have $k_{n}(t) / N_{n} \rightarrow 1$ as $n \rightarrow \infty$ for $0<t \leq 1$, so by Lemma 4 , $\varphi(\{p\}, 0) \rightarrow 1=\varphi(\emptyset, 0)$ as $p \rightarrow \infty$, that is, $(\{p\}, 0) \rightarrow(\emptyset, 0)$ in $T$. However, since $k_{n}(t)<N_{n}$ for all $n \in \mathrm{N}$ and $t \in[0,1]$ then $\mu(L)=1$ by Lemma 5, so $\mu_{*}\left(\widehat{T} \backslash T^{\prime}\right)=1$. For $0 \leq u<v \leq 1$ we have $k_{n}(v)>N_{n} e^{-e^{-n v}}-1$ and $k_{n}(u) \leq N_{n} e^{-e^{-n u}}$, so

$$
\frac{\log \left[N_{n} / k_{n}(v)\right]}{\log \left[N_{n} / k_{n}(u)\right]}<e^{n u} \log \frac{e^{e^{-n v}}}{1-e^{e^{-n v}} / N_{n}}<e^{-n(v-u)}-e^{n u} \log \left(1-\frac{e}{N_{n}}\right) \rightarrow 0 .
$$

By Theorem 3 it follows that $T$ is compact.

Define an equivalence relation $\sim$ in $T$ by the condition that $s \sim t$ if and only if $d(s, t)=0$, denote by $h(s)$ the equivalence class containing $s \in T$, and define $S=h(T)$. Then $S$ is a metric space under the quotient metric, also denoted by $d$. If $s \sim t$ and $u \sim v$ then $s \cup u \sim t \cup v$ by (4), so we can make $S$ a semilattice by defining $h(s) \cup h(u)=h(s \cup u)$ for $s, u \in T$. The inequality (4) carries over to $S$, which is therefore a topological semigroup. Since $T$ is compact, so is $S$. The inequality (5) shows that there is a unique function $\Phi$ on $S$ such that $\varphi=\Phi \circ h$. Clearly $\Phi$ is positive definite. The inequality (5) carries over to $S$ and shows that $\Phi$ is continuous. If $\lambda$ is the unique measure on $\widehat{S}$ such that $\Phi=\widehat{\lambda}$ then $\mu(A)=\lambda(\{\sigma \in \widehat{S} \mid \sigma \circ h \in A\})$ for $A \in \mathscr{B}(\widehat{T})$. In particular, $1=\mu(L)=\lambda(\{\sigma \in \widehat{S} \mid \sigma \circ h \in L\})$. Since the conditions $\sigma \in \widehat{S}$ and $\sigma \circ h \in L$ imply that $\sigma$ is discontinuous at 0 then $\lambda_{*}\left(\widehat{S} \backslash S^{\prime}\right)=1$.

Acknowledgments. Correspondence with Paul Ressel has been very helpful.

\section{REFERENCES}

1. C. Berg, Positive definite and related functions on semigroups, in: K. H. Hofmann, J. D. Lawson, and J. S. Pym (eds.), The Analytical and Topological Theory of Semigroups, Walter de Gruyter \& Co., Berlin 1990.

2. C. Berg, J. P. R. Christensen and P. Ressel, Harmonic Analysis on Semigroups, SpringerVerlag, New York 1984.

3. G. Choquet, Theory of capacities, Ann. Inst. Fourier (Grenoble) 5 (1954), 131-295.

4. R. J. Lindahl and P. H. Maserick, Positive-definite functions on involution semigroups, Duke Math. J. 38 (1971), 771-782. 
5. A. Povzner, Über positive Funktionen auf einer Abelschen Gruppe, Dokl. Akad. Nauk SSSR 28 (1940), 294-295.

6. D. A. Raikov, Positive definite functions on commutative groups with an invariant measure, Dokl. Akad. Nauk SSSR 28 (1940), 296-300.

7. P. Ressel, Bochner's theorem for finite-dimensional conelike semigroups, Math. Ann. 296 (1994), 431-440.

8. P. Ressel, private communication.

9. K. A. Ross, The structure of certain measure algebras, Pacific J. Math. 11 (1961), 723-737.

10. A. Weil, L'Intégration dans les Groupes Topologiques et ses Applications, Actualités Sci. Indust. 869, 1940.

NANDRUPSVEJ 7 ST. TH.

DK-2000 FREDERIKSBERG C

DENMARK

Email : torben.bisgaard@get2net.dk 